\title{
ASSESSMENT OF PARTICLE PACKING CHARACTERISTICS AT INTERFACES BY SPACE SYSTEM
}

\author{
PIET STROEVEN AND MARTIJN STROEVEN
}

Faculty of Civil Engineering and Geosciences, Stevinweg 1, 2600 GA, Delft, Netherlands

(Accepted April 19, 2000)

\begin{abstract}
Interest in material structure has been raised by upgrading of cementitious systems. Computer-simulation of material structure has been pursued so far by using random generators. Such systems were successfully applied for estimating global properties. Realistic estimation of structure-sensitive properties, however requires a more appropriate simulation of material structure. The SPACE (Software Package for the Assessment of Compositional Evolution) system has been developed for that purpose. It incorporates particle interaction and gravitational influences, which are typical for the 'natural' processes underlying the formation of material structure in the production stage. The capabilities are discussed of the two computersimulation methods for generating structural information relevant for either structure-insensitive, or structure-sensitive properties in bulk and in the interfacial transition zone (ITZ). The structural information will deal with composition and configuration of the material, respectively. The use of random generatorbased (RG) systems is demonstrated not justified when dealing with structure-sensitive problems.
\end{abstract}

Keywords: bond, computer simulation, interface, particle packing, random generator, SPACE system.

\section{INTRODUCTION}

A growing interest in material structure is resulting from efforts to upgrade concrete materials. Such efforts involve the addition of silica fume and other (super-)fine mineral admixtures to concrete, the blending of cements, or the increase in the range of particle sizes .of the aggregate. A realistic structuresimulation system would offer insight into the relevant features of the aggregation of particulate matter, ultimately even setting the scene for systematic optimization of such materials. Close to the mould, material structure on mesolevel should deviate from that in bulk. Surfaces of aggregate particles will similarly disturb the 'natural' processes of aggregation of matter on microlevel. Mostly, experimental approaches focus on compositional changes in the vicinity of interfaces. Structuresensitive properties of the material will depend, however, on material configuration.

Insight into material configuration is therefore a pre-requisite in understanding mechanical behaviour of such composites. This holds in particular for the post-peak range, where the non-uniformity of the packing is of greater significance than any regular property. The common way to simulate particulate composites is by using so called random generator (RG) systems. This approach was proven successful for simulating structural features relevant for global pre-peak concrete behaviour. Since major contributions to local strength in cementitous materials are due to van der Waals binding forces, the spacing of neighbouring particles will be of paramount importance. Moreover, once bond cracks have been formed at particle-matrix interfaces, the process of coalescence which ultimately will lead to fracture, will also be governed by (particle) spacing. So, for the estimation of such structure-sensitive phenomena, the nearest neighbour spacing should be realistically simulated. The differences in output of such a 'classical' approach and one by the recently developed 'dynamic' SPACE (Software Package for the Assessment of Compositional Evolution) system pursued in this paper

\section{COMPUTER SIMULATION OF GRANULAR MATERIALS}

The granular material structure is represented in both computer simulation approaches by a set of distinct elements. Each element corresponds to a distinguishable, characteristic phase in the material. For example, concrete is modelled as a set of elements representing the aggregate particles. The elements are dispersed in a presumably homogeneous mortar matrix moulded in a container. The parameters 
describing the static conditions of the internal structure are the locations, orientations, and shapes of the various individual elements. Since the elements represent real physical phases in the material, physical properties can be assigned to each element along with its shape and size, at least in principle. The most difficult task, however, is the derivation of the location and the orientation of these nonoverlapping elements.

In a static simulation system, the particles are sequentially located inside a container. Each location is governed by randomly generated coordinates. 'Overlap' with earlier generated particles will result in rejection, whereupon the generation process is continued. The number of rejections will already increase dramatically at moderate volume fractions, making the process of generation very timeconsuming. Also, simulation of a multi-size particle composite requires starting with the largest particles in the mix. But maximum particle densities met in concrete technology (volume contents of $70 \%$ or higher) cannot be realized even under such conditions. The system obviously excludes the mechanism of particle interaction, which is so characteristic for the production stage of cementitious materials. Three of the more popular systems in this category were developed by Roelfstra (1989), by Diekkamper (1984), and by Bentz et al. (1993).

The SPACE system is based on a dynamic concept of simulating the production process of a composite material; i.e., in case of concrete: mixing of aggregates and paste, or cement and water. The details of the generation system have been published by the Stroeven and Stroeven (1996). To be able to simulate effects such as clustering and to reach high volume densities, element motion and inter-element collision are modelled, leading to effective ingredient mixing. This stage is referred to as the dynamic stage. The final distribution state can incorporate effects due to gravity and to inter-particle influences. Simple physical laws may be used to define the inter-particle relationships, by introducing parameters such as specific mass and energy dissipation. High packing densities can be obtained, representing dense random packing states, typical of aggregate and binder particles in cementitious materials.

\section{ESTIMATION OF MATERIAL BEHAVIOUR}

The first illustrative example concerns a study of the packing density of multi-sized, spherical cement grains in the neighbourhood of a rigid boundary. Hence, this study deals with the compositional discontinnity in the packing of binder particle in the so called interfacial transition zone (ITZ). Such data could underly estimates of structucre-insensitive properties. The particle size distribution of Portland cement particles is taken in accordance with the Rosin-Rammler cumulative particle size distribution function, $G(d)=1-\exp \left(-b d^{n}\right)$. A mixture was simulated by both systems (RG and SPACE) representing a water to cement ratio of 0.5 . Results demonstrated clearly that the compositional differences between the two systems were not dramatic. 30,000 particles between 1 and $20 \mu \mathrm{m}$ were considered for parameter settings of $n=1.003$ and $b=0.0288$. Fig. 1 shows an additional simulation at a water to cement ratios of 0.17 by SPACE. Simulation by the RG system of such densely packed cement grains systems would be very complicated. Total volume fraction of the $1-20 \mu \mathrm{m}$ cement particles is plotted as function of the distance to the interface.

A popular way to study effects of packing density differences in the ITZ on mechanical properties is by microhardness measurements. If it is assumed for the present purpose that 'hardness' is similar for all cement particles, then outcomes of microhardness tests can be associated with volume fraction of particles. It should be emphasized that the Knoop indentor yields an average value over its width, $b$, in a direction perpendicular to the interface. With the high hardness value of the aggregate particle influencing the outcomes for small distances to the interface, $r \leq b / 2$, a through-type of micro-hardness curve can be derived from both sets of simulation data of Fig. 1. Such microhardness characteristics are reported in the relevant literature (e.g. Wang, 1988). Generalizing, either one of the systems can be employed for estimating structure-insensitive properties, such a micro-hardness. It must be obvious, though, that the averaging character of the experimental data on microhardness will lead to biased (too large) estimates for the thickness of the ITZ. In comparing simulation results and experimental data it should be recognized, moreover, that experimental measurements performed on a set of serial sections will always be biased (too large). When $\bar{\mu}$ is the average measured thickness of the ITZ, and $t$ the real thickness, it can easily be seen that $\bar{\mu}=t \cdot \overline{\sin ^{-1} \alpha}=\pi / 2$. Here, $2 \alpha$ is the angle at the particle's center under which the circular section plane can be viewed. This holds for a practical range of aggregate grain sizes, whereby $t \ll d(d=$ grain 
size). Finally, it should be noted that a conventional cement will contain a measurable quantity of $80 \mu \mathrm{m}$ grains, while the example of Fig. 1 is limited to the 1$20 \mu \mathrm{m}$ range. When all those factors are considered, it could be concluded that experimental observations of the ITZ's thickness would satisfactorily match the simulated ones, which are solely based on the so called packing phenomenon. Of course, estimates and experiments should focus on properties of similar structure-sensitivity.

The particle fractions underlying the total volume curve in Fig. 1 reveal a typical size-dependent behaviour away from the interface, causing a longrange discontinuity in grading. The fines concentrate near the interface. They govern primarily the steep increase in total volume of particles near the interface. The fraction of the largest particles has its peak in volume density at a distance correlated with particle size. With a higher water to cement ratio - as in the present case - this distance is close to the average particle radius, but for the lower water to cement ratios used in high performace concretes, this distance increases: particles seem to be pushed further inwards (Stroeven, 1999). So, when the thickness of the ITZ is based on grading homogeneity, it will significantly exceed that based on composition. The same will hold for the homogeneity in medium structure-sensitive properties depending on grading. This is visualized in Fig. 2, where the mean free path $\lambda$, as introduced by Fullman (1953), is used. The plotted parameter, $\lambda^{-3}$, can be interpreted as proportional to an overal bonding capacity relying on van der Waals forces (Wittmann, 1971). Characteristic differences between the two systems were visualized at lower w/c ratios, but the RG systems fail in simulating interface packing situations in such high performance concrete mixes.

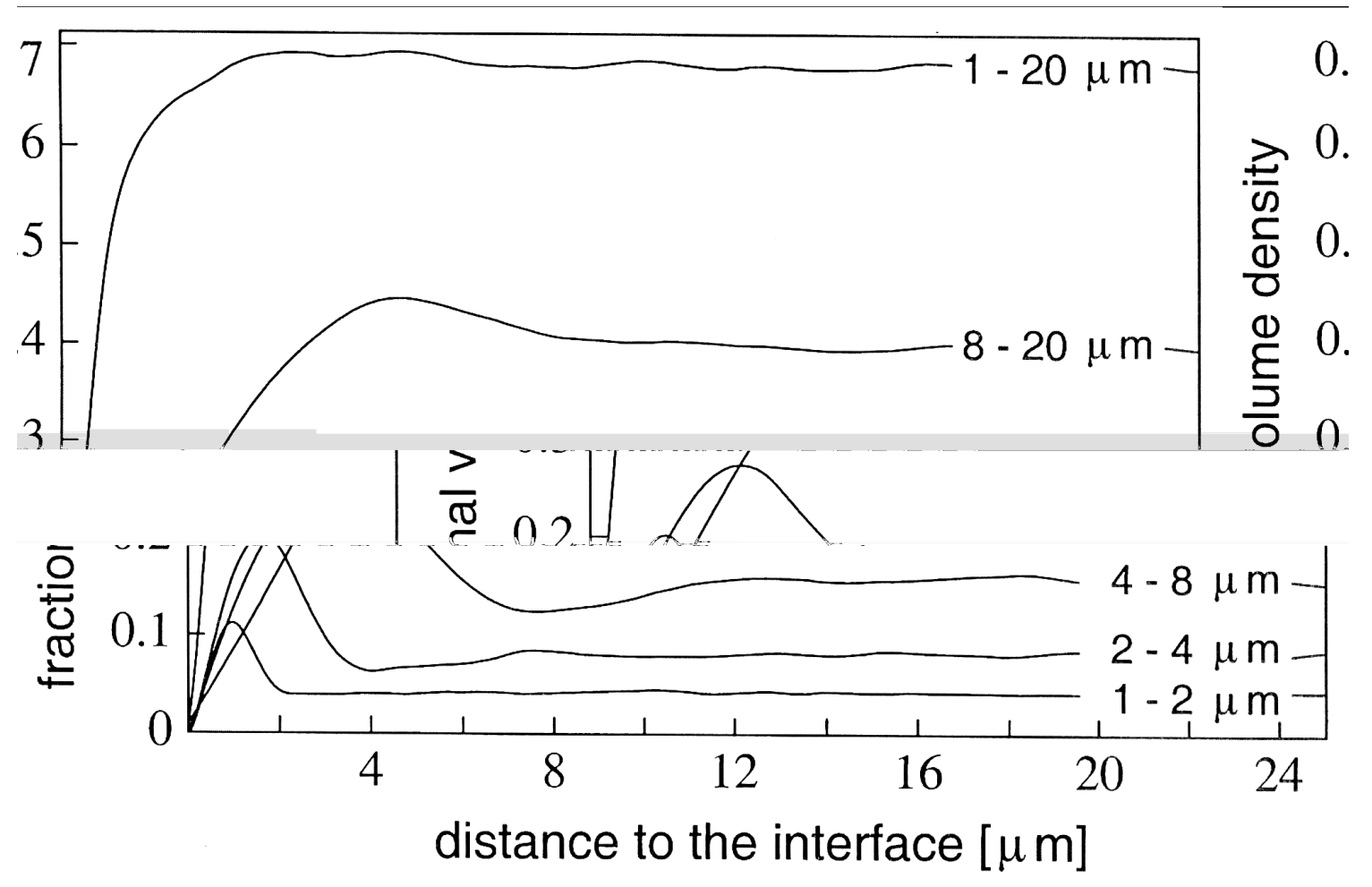

Fig. 1. Changes in volume fraction of distinct size ranges and in total volume fraction of cement particles in the ITZ in concrete. 


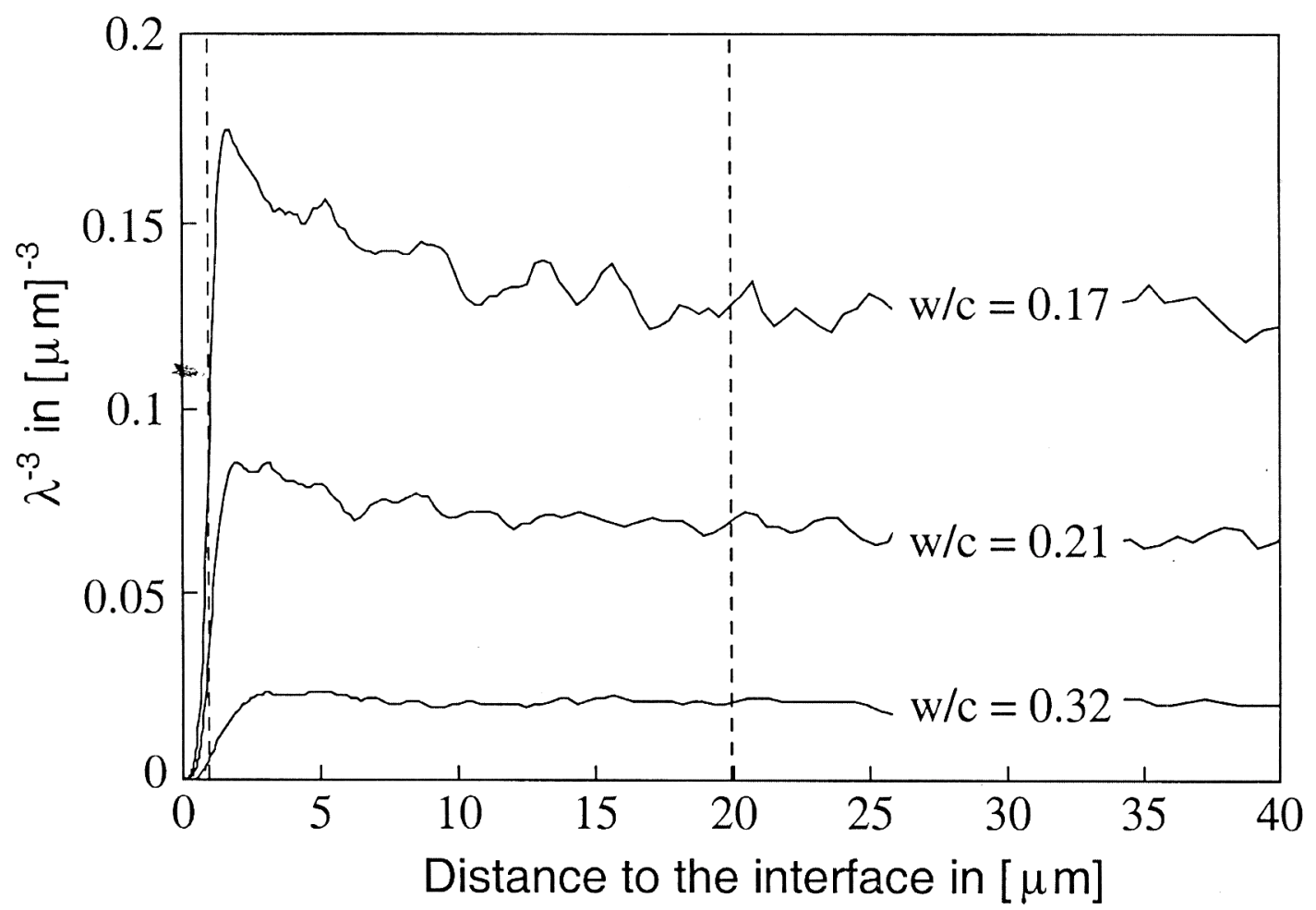

Fig. 2. Normalized global inter-particle bond capacity in the ITZ, expressed by $\lambda^{-3}$, as function of the distance to the interface. Dashed lines indicate size range of particles. For $w / c=0.17$ the ITZ extends to $20 \mu \mathrm{m}$, approximately.

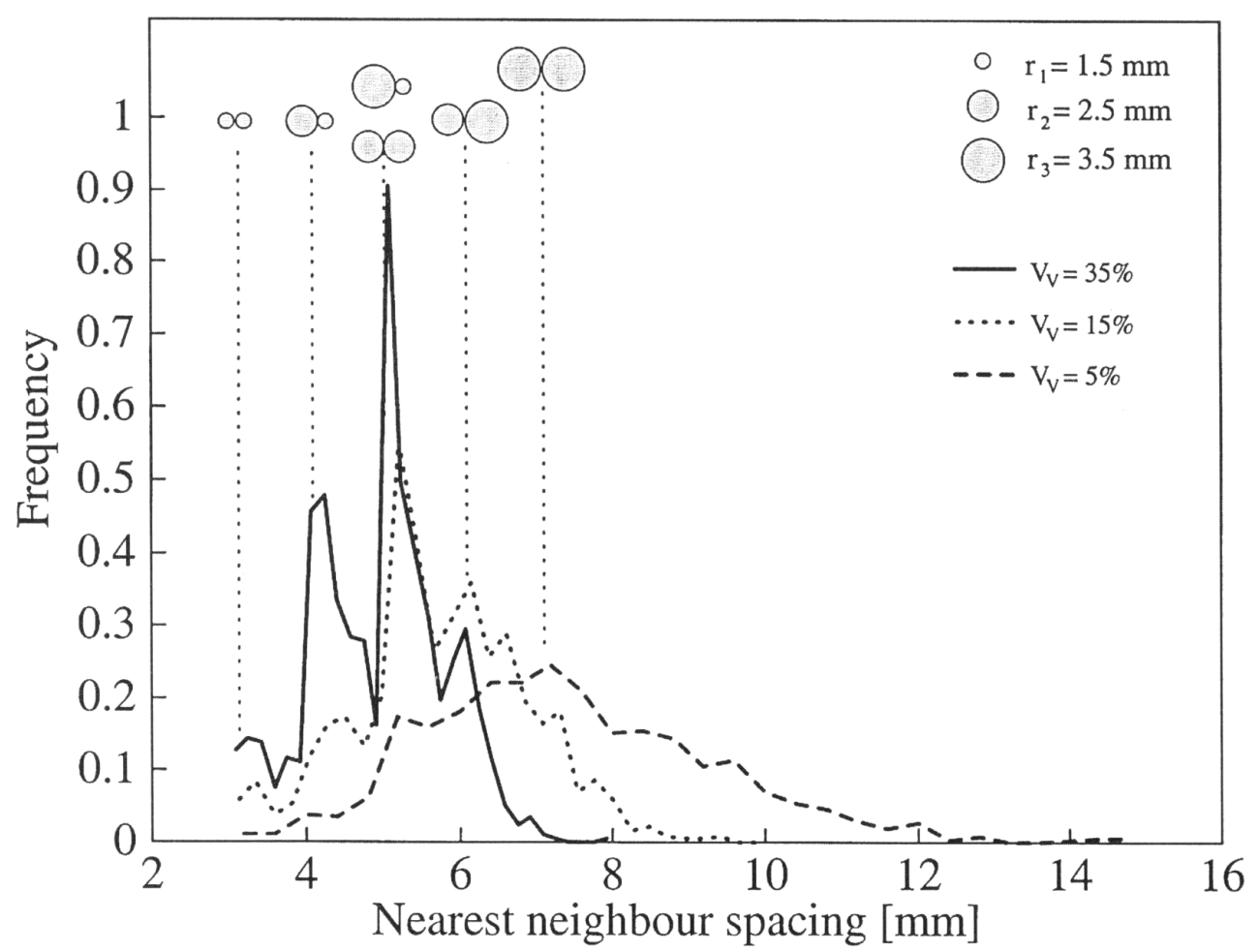

Fig. 3. Nearest neighbour distance distributions for aggregate mixture composed of three particle sizes and a total volume content of 5,15 and $35 \%$, respectively. 
The most relevant configuration parameter is probably the nearest neighbour distance. This parameter allows the assessment of the respective capabilities of the two structural simulation systems when studying structure-sensitive properties like crack initiation. Initiation of cracking will be mainly at interfaces between aggregate particles in concrete. It will be the result of stresses exceeding local (physical) bond capacity. Van der Waals bonding forces will be governed by surface-to-surface spacing of cement particles, or of the cement particles and the aggregrate grain interface. The nearest neighbour distance is intimately related to surface spacing, so it can be used for the present purpose. The rejection of particle 'overlap' leads in the RG-based simulations to more evenly distributed particles than met in nature, inevitably revealing a natural phenomenon as particle clustering to be suppressed. Conclusive evidence for these statements Fig. 3 in Stroeven and Stroeven (1996) convincingly demonstrates the fastly growing gap between the two structural simulation concepts in estimating the nearest neighbour distance distribution at increasing volume densities. Data confirming the capability of the SPACE system to simulate the natural process of particle clustering is presented Fig. 3. A mixture of three different particle sizes $\left(d_{1}=3, d_{2}=5 \mathrm{~mm}\right.$ and $\left.d_{3}=7 \mathrm{~mm}\right)$ is generated by the SPACE system for increasing total volume fractions, $V_{V}$, of the aggregate. A strong tendency for particle clustering when particle density increases is reflected by the distinct peaks in the nearest neighbour distance distribution curve for the highest density case $(35 \%)$. The peaks correspond to zero surface-to-surface spacings of the different combinations of particle sizes. The proper way in which the SPACE system realistically generates small nearest neighbour distances (thereby revealing particle clustering) is a prerequisite for developing reliably estimates for structure-sensitive properties (such as interparticle bond and aggregate-cement bonding).

\section{CONCLUSIONS}

Computer-simulation allows for accurate assessment of compositional parameters characterizing particulate structures in cementitious materials, either on mesolevel where aggregate grains are packed in a container, or on microlevel where (blended) Portland cement particles are dispersed in pockets between these aggregate grains. Bulk properties, or discontinuities near boundaries, can be studied in this way. Compositional parameters such as volume percentage of particles, or porosity should be used in connection with structure-insensitive properties. The paper indicates that microhardness properties in the ITZ could be estimated properly, at least qualitatively, in this way. Such compositional problem can be approached by random generator-based systems and by the SPACE system alike.

RG-based systems generate more evenly distributed particles, thereby under-estimating the natural phenomenon of particle clustering. The nearest neighbour distance distribution in moderately to densely packed particulate structures (as met in cementitious materials) simulated by RG systems, will be significantly biased, as a result. Hence, material configuration in such dense random packings will be poorly represented by RG-based simulation data. Structure-sensitive properties based on particle packing, like van der Waals bond between densely packed hydrated Portland cement particles, or between such particles and the interface, can be expected only properly simulated on the basis of SPACE-generated particulate structures.

A preliminary report of some of the data was presented at the $X^{\text {th }}$ International Congress for Stereology, Melbourne, Australia, 1-4 November 1999.

\section{REFERENCES}

Bentz DP, Garboczi EJ, Stutzman PE (1993). Computer modelling of the interfacial zone in concrete. In: Maso JC (ed). Interfaces in Cementitious Composites. London: E \& FN Spon, 107-16.

Diekkämper R (1984). Ein Verfahren zur numerischen Simulation des Bruch- und Verformungsverhaltens spröder Werkstoffe. Techn. Wissenschaftliche Mitteilungen der Inst für Konstruktiven Ingenieursbau. Ruhr Univ Bochum, 84(7).

Fullman RL (1953). Measurement of particle sizes in opaque bodies. Trans Met Soc AIME 197(March):447-52.

Roelfstra PE (1989). A numerical approach to investigate the properties of numerical concrete, $\mathrm{PhD}$ Thesis, EPFL-Lausanne.

Stroeven M, Stroeven P (1996). Computer-simulated Internal Structure of Materials. Acta Stereol 15(3):247-52.

Stroeven M (1999). Discrete model for the structure assessment of composite materials, PhD Thesis, Delft Univ Technology.

Stroeven M, Stroeven P (1999). Computer-simulation of particle packing in cementitious systems. In: Malhotra VM, Helene P, Prudêncio LR, Dal Molin DCC, eds. HPC and Performance and Quality of Concrete Structures. Farmington Hills: ACI, 327-40. 
Wang Y (1988). The effect of bond characteristics between steel slag fine aggregate and cement paste on mechanical properties of concrete and mortar. In: Mindess S, Shah SP, eds. Bonding in Cementitious Composites. MRS Symp Proc 114:49-54.
Wittmann F (1971). Experiments to determine van der Waals forces. In: Te'eni M, ed. Structure, Solid Mechanics and Engineering Design. London: Wiley, 295-300. 